\title{
Impact of community structure on information transfer
}

\author{
Leon Danon, ${ }^{1,2}$ Alex Arenas, ${ }^{2}$ and Albert Díaz-Guilera ${ }^{1}$ \\ ${ }^{1}$ Departament de Física Fonamental, Universitat de Barcelona, Marti i Franques 1, 08028 Barcelona, Spain \\ ${ }^{2}$ Departament d'Enginyeria Informàtica i Matemàtiques, Campus Sescelades, Universitat Rovira i Virgili, 43007 Tarragona, Spain
}

(Received 14 September 2007; published 3 March 2008; publisher error corrected 12 March 2008)

\begin{abstract}
The observation that real complex networks have internal structure has important implication for dynamic processes occurring on such topologies. Here we investigate the impact of community structure on a model of information transfer able to deal with both search and congestion simultaneously. We show that networks with fuzzy community structure are more efficient in terms of packet delivery than those with pronounced community structure. We also propose an alternative packet routing algorithm which takes advantage of the knowledge of communities to improve information transfer and show that in the context of the model an intermediate level of community structure is optimal. Finally, we show that in a hierarchical network setting, providing knowledge of communities at the level of highest modularity will improve network capacity by the largest amount.
\end{abstract}

DOI: 10.1103/PhysRevE.77.036103

\section{INTRODUCTION}

The continuing intensity that accompanies the study of complex networks has led to many important contributions in a variety of scientific disciplines (for a recent review see [1]). Specifically, the study of transport properties of networks is becoming increasingly important due to the constantly growing amount of information and commodities being transferred through them. A particular focus of these studies is how to make the capacity of the network maximal while minimizing the delivery time. Both network packet routing strategies and network topology play essential parts in traffic flow in networks.

Traditionally routing strategies have been based on the idea of maintaining routing tables of the best approximation of the shortest paths between nodes. In realistic settings, however, the knowledge that any one of the nodes has about the topology of the network will be incomplete. So, much of the focus in recent studies has been on searchability. In particular, distributed search using only local information has been shown to be efficient in spatially embedded networks $[2,3]$. Networks with scale-free degree distributions are particularly navigable using local search strategies due to the presence of highly connected hubs [4].

However, when the number of search problems the network is trying to solve increases, it raises the problem of congestion at central nodes. It has been observed, both in real world networks [5] and in model communication networks [6-12], that the networks collapse when the load is above a certain threshold and the observed transition can be related to the appearance of the $1 / f$ spectrum of the fluctuations in Internet flow data $[13,14]$.

These two problems, search and congestion, that have so far been analyzed separately in the literature can be incorporated in the same communication model. Previous work has contributed a collection of models that capture the essential features of communication processes and are able to handle these two important issues simultaneously $[8,10,15,16]$. In these models, agents are nodes of a network and can interchange information packets along links in the network. Each agent has a certain capability that decreases as the number of packets to deliver increases. The transition from a free phase to a congested phase has been studied for different network
PACS number(s): 89.75.Hc, 87.23.Ge

architectures in $[8,10]$, whereas in [15] the cost of maintaining communication channels was considered.

The topology of the network also plays a central part in communication processes. In [16] the problem of finding optimal network topologies for both search and congestion for a fixed number of nodes and links was tackled. It was found that in the free regime, highly centralized topologies facilitating search are optimal, whereas in the congested regime decentralized topologies which distribute the packet load between nodes are favored. It has been shown that shortest path routing algorithms are not optimal for scale-free networks due to the presence of communication bottlenecks [17] and several alternative routing strategies have been proposed to take advantage of the scale-free nature of complex networks [11,18-22].

On the other hand, many networks found in nature have been observed to have a modular or community structure. Communities are those subsets of nodes that are more densely linked internally than to the rest of the network. Identifying communities in networks has become a problem which has been tackled by many researchers in recent years (see, for example, Refs. [23-25], and for reviews see $[26,27])$. Furthermore, communities are often organized in a hierarchical way $[16,28-31]$. That is, large communities are often comprised of several smaller communities. Despite all these efforts, the impact that community structure has on information transfer has not been considered.

The aim of this paper is twofold: First, we will investigate the effect that community structure has on the model of search and congestion, and second we will propose an alternative routing strategy and demonstrate its impact in the presence of community structure. In the next section we will describe the model and recall the most important analytical results. In Sec. II we will consider the effect that a modular structure of varying strength has on the behavior of the model. We will then show how knowledge of this community structure can be taken advantage of to improve transport processes in networks. In the final section, we give some concluding remarks.

\section{COMMUNICATION MODEL}

The communication model considers that the information flowing through the networks is formed by discrete packets 
sent from an origin node to a destination node. Each node is an independent agent that can store as many packets as necessary. However, to have a realistic picture of communication we must assume that the nodes have a finite capacity to process and deliver packets. That is, a node will take longer to deliver two packets than just one. A particularly simple example of this would be to assume that nodes are able to deliver one (or any constant number) information packet per time step independent of their load, as in the model of decentralized information processing in firms of Radner [32] and in simple models of computer queues $[6,7,9,15]$, but note that many alternative situations are possible.

In the present model, each node has a certain ability to deliver packets which is limited. This limitation in the ability of agents to deliver information can result in congestion of the network. When the amount of information is too large, agents are not able to handle all the packets and some of them remain undelivered for extremely long periods of time. The maximum amount of information that a network can manage before collapse gives a measure of the quality of its organizational structure. In this study, the interest is focused on when congestion occurs depending on the topology of the network [15].

The dynamics of the model is as follows. At each time step $t$, an information packet is created at every node with probability $\rho$. Therefore $\rho$ is the control parameter: Small values of $\rho$ correspond to low density of packets and high values of $\rho$ correspond to high density of packets. When a new packet is created, a destination node, different from the origin, is chosen randomly in the network. Thus, during the following time steps $t+1, t+2, \ldots, t+T$, the packet travels toward its destination. Once the packet reaches the destination node, it is delivered and disappears from the network.

The time that a packet remains in the network is related not only to the distance between the source and the target nodes, but also to the amount of packets in its path. Nodes with high loads-i.e., high quantities of accumulated packets-will take longer to deliver packets or, in other words, it will take more time steps for packets to cross regions of the network that are highly congested. In particular, at each time step, all the packets move from their current position, $i$, to the next node in their path, $j$, with a probability $p_{i j}$. This probability $p_{i j}$ is called the quality of the channel between $i$ and $j$. In this paper, we take the special case that each node is able to send one packet at each time step. It is important to note, however, that the model is not deterministic. Here, a packet which is waiting at a particular node, will be sent with equal probability as any other packet waiting at the same node.

The packets in the present model have a limited radius of knowledge, that is, they are able to determine whether a node within a certain distance $r$ is the destination node. In this case, the packet takes the shortest possible route to the destination, otherwise, it travels down a link chosen at random. In this paper we set $r=1$, so that only nearest neighbors are recognized. It has been shown in previous work that in the free phase, there is no accumulation at any node in the network and the number of packets that arrive at node $j$ is, on average, $\rho B_{j} /(S-1)$, where $B_{j}$ is the effective betweenness of node $j$ which is defined as the fractional number of paths that packets take through node $j$ and $S$ is the number of nodes in the network. A particular node will collapse when $\rho B_{j} /$ $(S-1)>1$ and the critical congestion point of the network will be

$$
\rho_{c}=\frac{S-1}{B^{*}},
$$

where $B^{*}$ is the maximum effective betweenness in the network, that corresponds to the most central node.

If the routing algorithm is Markovian, which is the case here, it is possible to estimate $B_{j}$ analytically. The search and congestion process can be formulated as a Markov chain, which is dependent on the packet transition probability matrix. This matrix is derived from the adjacency matrix of the network, the radius of knowledge $r$, and the search algorithm. Using this formulation, $B_{j}$ of each node can be calculated analytically for any $r$ [10]. In these cases, the paths that the packets take will not be the shortest paths. As the radius of knowledge increases, $B_{j}$ converges to shortest path betweenness and will be equal to it when $r$ is greater or equal to the diameter of the network.

\section{PACKET DYNAMICS OF COMMUNICATION MODEL ON NETWORKS WITH COMMUNITY STRUCTURE}

The model from [10] can be further exploited to look at the effects that community structure has on dynamics. To this end we need to be able to construct networks with controllable community structure. We choose to use a family of pseudorandom networks since all other properties (such as node degree and clustering) will be equivalent to fully random networks. The only thing that we will vary is the strength of community structure.

First we employ the networks proposed in [33]. These networks are comprised of 128 nodes which are split into four communities of 32 nodes each. Pairs of nodes belonging to the same community are linked with probability $p_{\text {in }}$, whereas pairs belonging to different communities are joined with probability $p_{\text {out }}$. The value of $p_{\text {in }}$ is chosen so that the average number of links a node has to its own community, $Z_{\text {in }}$, can be controlled. While $p_{\text {in }}$ (and therefore $Z_{\text {in }}$ ) is varied freely, the value of $p_{\text {out }}$ is chosen to keep the total average node degree, $k$, constant, and set to 16 . As $Z_{\text {in }}$ is increased from zero, the communities become better defined and easier to identify.

To address the question of hierarchical structure we use a generalization of the model of generation of networks with community structure that includes two hierarchical levels of communities as introduced in [28]. The graphs are generated as follows: In a set of 256 nodes, 16 compartments are prescribed that will represent our first community organizational level. Each of these subcommunities contains 16 nodes each. Furthermore, four second level communities are prescribed, each containing four subcommunities, that is 64 nodes each. The internal degree of nodes at the first level $Z_{\mathrm{in}_{1}}$ and the internal degree of nodes at the second level $Z_{\mathrm{in}_{2}}$ are constrained to keep an average degree $Z_{\mathrm{in}_{1}}+Z_{\mathrm{in}_{2}}+Z_{\text {out }}=18$. From 
now on, networks with two hierarchical levels are indicated as $Z_{\mathrm{in}_{1}}-Z_{\mathrm{in}_{2}}$, e.g., a network with 13-4 means 13 links with the nodes of its first hierarchical level community (more internal), four links with the rest of the communities that form the second hierarchical level (more external) and one link with any community of the rest of the network.

As a simple measure of structural efficiency of the network in terms of packet transport, we can consider the number of packets present in the network. We allow the dynamics to reach a steady state, which we detect by considering the rate at which the number of packets increases in the system. Once this rate becomes small, fluctuating around 0 , we have reached the end of the transient. It is important to note that when $\rho>\rho_{c}$ the system never reaches a steady state, the mean number of packets keeps growing linearly with time, and the rate never becomes very small. We also average over several realizations, since the number of packets in the system is subject to statistical fluctuations.

\section{A. Original communication model}

First, we simulate the dynamics of the model described above, in which the packets have no knowledge of the topology of the network at the level of community structure. Introducing community structure in the network topology over which the dynamics occur increases the traffic load on the nodes which connect communities. This is in agreement with the finding that cutting links with the highest betweenness separates communities [23]. It follows that the effective betweenness of the nodes at each end of the bridge links will also be increased. As a result, the capacity of the network to deliver packets is reduced in function of how fuzzy the community structure is.

From Fig. 1 we can see that the analytical calculation from Sec. II, of the onset of congestion $\rho_{c}$ agrees very well with the point at which the number of floating packets diverges. As the strength of community structure is increased by raising $Z_{\text {in }}, \rho_{c}$ is reduced. This seems logical, since the origin and destination of packets are chosen at random. It follows that the probability of creating a packet with both origin and destination in one community is $1 / 4$. All other packets will necessarily have to pass through at least one central "bridge" node that connects two communities. This leads to an increase in the number of packets that pass through bridge nodes, increasing its effective betweenness. As a result of receiving a disproportionate amount of packets, these nodes will collapse at lower values of $\rho$, leading to a cascade of collapses throughout the network. This effect becomes more and more pronounced as $Z_{\text {in }}$ increases, so, the stronger the community structure, the lower $\rho_{c}$.

In the case of hierarchical networks, we concentrate on three different network topologies which are particularly instructive, 13-4, 14-3, and 15-2. Once again the analytical calculation corresponds very well to the point at which the number of floating packets diverges, see Fig. 2. It is worth noting that these three networks have almost the same $\rho_{c}$. This is due to the fact that the average number of links per node between communities of size 64 is constant and set to 1. What is varied is the strength of the intermediate and
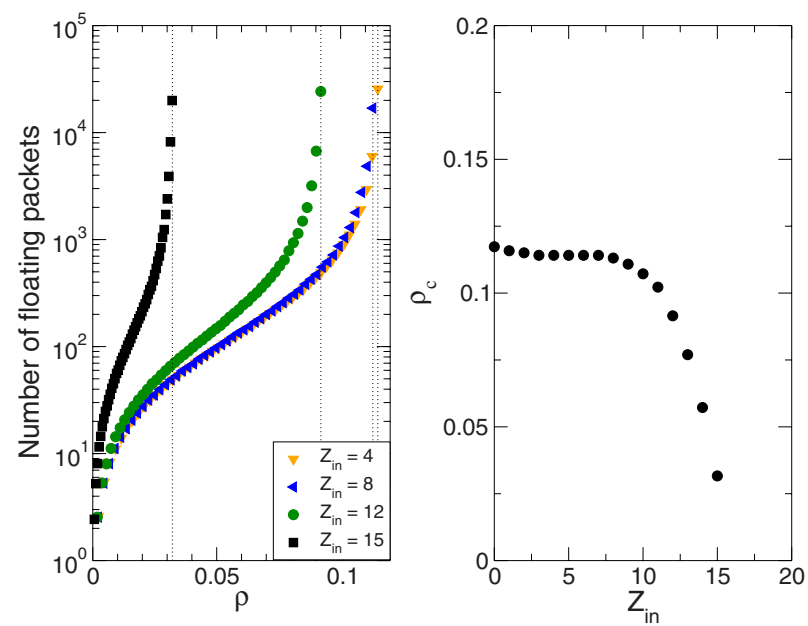

FIG. 1. (Color online) Each point represents the number of packets averaged over 100 realizations in the steady state of the dynamics. (a) Number of floating packets as a function of $\rho$ using the original search algorithm in networks with one level of community structure. The different colors denote varying levels of community strength as controlled by the parameter $Z_{\text {in }}$ and the vertical lines correspond to the analytical prediction of the onset of congestion [Sec. II, Eq. (1)]. (b) Onset of congestion $\rho_{c}$ for varying $Z_{\text {in }}$.

innermost level of community structure. In the case of the original communication model, this shows little effect.

\section{B. Modifying the communication model}

Clearly, networks with strong community structure are less efficient at delivering packets which are oblivious to the underlying topology. But, what happens when we give the search process some information about the community structure? To address this question we propose a simple modification of the way packets are transferred between nodes.

Let us consider a packet generated at node $i$ in community $c_{i}$ with destination node $j$ in community $c_{j}$. At each step in its path, the packet is given information of the community of neighboring nodes. Should the packet destination community be the same as that of any of the neighbors of the node that is processing the packet, the packet is sent to one of those

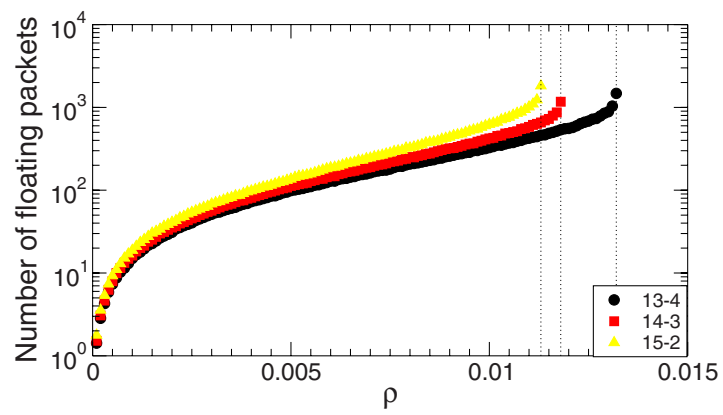

FIG. 2. (Color online) Number of floating packets as a function of $\rho$ using the original search in networks with hierarchical community structure on two levels. The vertical lines correspond to analytical prediction of the onset of congestion. 

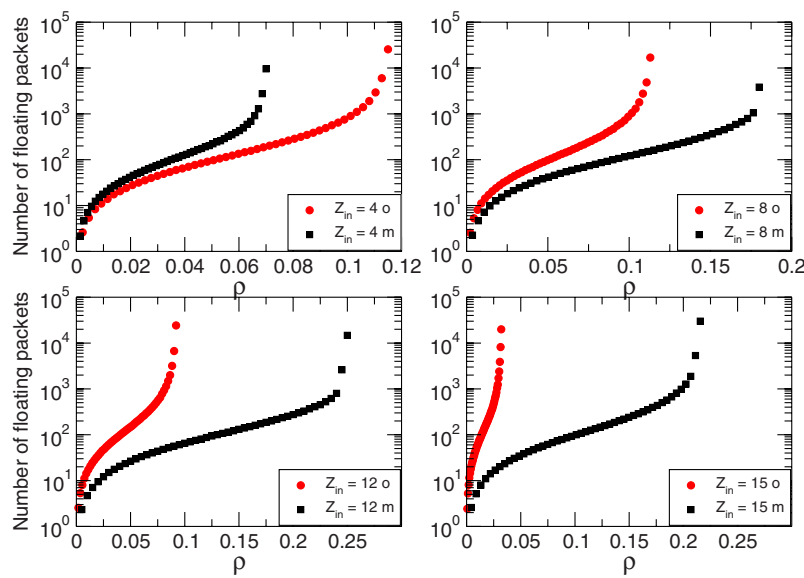

FIG. 3. (Color online) Comparison of the original search algorithm with the modified search algorithm for the same networks with community structure. The number of floating packets is plotted against $\rho$. The four panels depict four networks with varying community strength controlled by the parameter $Z_{\text {in }}$, and the red points show the original search algorithm and the black points denote the modified algorithm incorporating community information.

neighbors, otherwise it is sent down a link chosen at random. In this way, packets are able to arrive at the destination community without necessarily arriving at the destination node. The idea is that once within the destination community, finding the destination node is easier.

In Fig. 3 we plot the number of floating packets in the network at the steady state against the packet injection rate $\rho$. The dynamics are performed on networks with ad-hoc community structure of varying strength, controlled by the parameter $Z_{\text {in }}$, the average number of links internal to the community. When $Z_{\text {in }}=4$ the network is equivalent to an ErdösRenyi random graph with 128 nodes and 16 links per node. In this scenario, the original search algorithm performs much better in terms of ability to deliver packets. This seems logical: Giving packets information about communities which are not present will not improve the packet's ability to find the destination node. Indeed, for lower values of $Z_{\text {in }}$, this information is detrimental to efficiency, since the predefined partitions of the network actually contain fewer internal links, compared to external ones. In this scenario, packets are often sent to regions of the network which are less likely to contain the destination node. This is highlighted in Fig. 4(b), where we see that for very low values of $Z_{\text {in }}$ the original search algorithm collapses the network at much higher values of $\rho$ than the modified algorithm.

When the strength of the community structure is increased, the modified search algorithm improves the efficiency of the network considerably. For $Z_{\text {in }}>8$ [35], the onset of congestion in terms of $\rho$ is considerably higher for the modified search algorithm, and the same network is much more efficient at delivering packets for all values of $Z_{\text {in }}>8$. In other words, the modified algorithm is able to find more efficient routes to deliver packets and the network is able to handle a much higher load.

In the modified search algorithm, the calculation from Sec. II [Eq. (1)] is still valid, however, the analytic calcula-
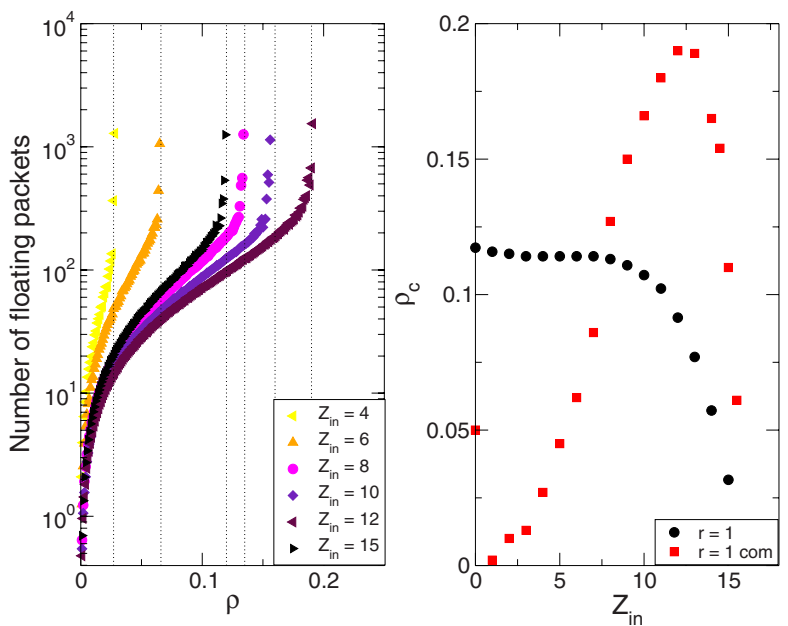

FIG. 4. (Color online) Onset of collapse for the modified search algorithm as applied to networks with community structure at a single level. (a) Number of floating packets as a function of $\rho$ for the modified search. Different colors denote varying community strengths, controlled by parameter $Z_{\text {in }}$ and the vertical lines now denote the estimate of the onset of congestion $\rho_{c}$. (b) $\rho_{c}$ as a function of internal connectivity $Z_{\text {in }}$ for both the original model (denoted $r=1)$ and the modified model (denoted $r=1 \mathrm{com}$ ).

tion of $B^{*}$ is more involved than in [10]. Nevertheless we can estimate $\rho_{c}$ of the network by looking at the point where the number of floating packets diverges. In Fig. 4(a), $\rho_{c}$ is estimated in this fashion. When the communities are extremely well defined, say $Z_{\text {in }}=15$, flow through the network is restricted. So even though the search method of the packets is greatly improved, and they are able to find the correct community in a short number of steps, flow is restricted by the formation of bottlenecks at the interface between two communities. It emerges that an intermediate community structure strength, $Z_{\text {in }}=12$ shows optimal efficiency in terms of $\rho_{c}$. This suggests that for the flow to be optimal there must be a balance between internal strength of communities and connections to other communities.

For the case of networks with hierarchical community structure as described above, community information can now be given at two levels. The packets can be given information about the community structure on the first level, that is, they are given knowledge about which community of the four communities of size 64 the destination node belongs to. From here on, this is denoted as $i=4$. Alternatively, we can give nodes information on the second level of community structure, so that packets know which one of the 16 communities of size 16 the destination node belongs to, which we denote $i=16$.

Once information about community structure is given to the packets, the efficiency of the network to deliver these is increased considerably as in the case of single level community structure. The level of community information which increases the efficacy of information flow by the largest amount is dependent on the topology of the network. Compared with no community information being given to the packets, $i=16$ increases the values of $\rho_{c}$ almost fivefold, in all three networks. In the case of $13-4, \rho_{c}$ is increased from 

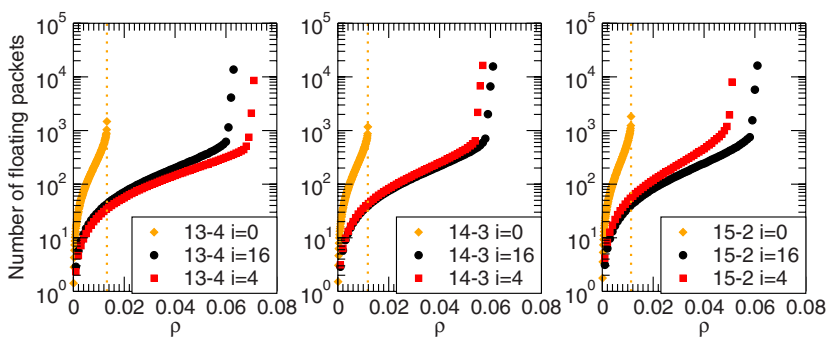

FIG. 5. (Color online) Number of floating packets in networks with hierarchical community structure. The number of floating packets is shown as a function of $\rho$ for three slightly different hierarchical networks, (a) 13-4, (b) 14-3, and (c) 15-2. For each, the level of community hierarchy information packets are given as varied between no information given $i=0$, information given at the first level $i=4$, and information given at the second level $i=16$. The vertical lines represent the analytical calculation as in Sec. II for the original search algorithm.

0.0132 to 0.064 . A stronger community structure at the second level, 14-3 and 15-2 does not make much of an impact when $i=16$, with $\rho_{c}$ being 0.063 for both.

However, when information is given at the intermediate level of community structure, $i=4$, the differences become more apparent. For the 13-4 configuration, community information at this level favors information diffusion more, with $\rho_{c}$ being 0.071 , higher than in the $i=16$ case. However in the case of the 14-3 network, the opposite is true: giving information at the alternative, $i=16$ level is (marginally) more beneficial. For the 15-2 network, giving more precise information causes a considerable improvement (see Fig. 5).

It is interesting to compare these results with other topological characterizations of complex networks. In particular, the most common measure related to community structure is the modularity measure, $Q$, proposed in [33] which measures the quality of a particular partition of a particular network. It is defined as follows:

$$
Q=\sum_{i}\left(e_{i i}-a_{i}^{2}\right),
$$

where the element $i, j$ of the matrix e represents the fraction of links between communities $i$ and $j$ and $a_{i}=\sum_{j} e_{i j}$. This value can also be measured at two levels. One is at the first level of the hierarchy, where nodes are grouped in four communities of 64 nodes each, which corresponds to the $i=4$ case. The other, corresponding to the $i=16$ case, is considering that the nodes are grouped in 16 communities of 16 nodes each. In the three networks we are considering, we only vary the strength of the second level of community structure, so for the $i=4$ case, the value of modularity remains constant. For the $i=16$ case however, the value of $Q$ varies with the strength of the second level. For the 13-4 network, the first level of community structure is a better partition in terms of $Q$, whereas for 14-3 and 15-2 the second level is a better partition. See Table I for values.

For 13-4, where the best partition is found at the first level of community structure $i=4$, giving packets information about the same level improves the efficiency of the network more than giving information at the second level. For 14-3
TABLE I. Table of values of the onset of collapse $\rho_{c}$ in hierarchical networks and the values of modularity of the same for two levels of grouping.

\begin{tabular}{lccccccc}
\hline \hline & \multicolumn{3}{c}{$\rho_{c}$} & & \multicolumn{2}{c}{$Q$} \\
\cline { 2 - 3 } \cline { 6 - 7 } Network & $i=0$ & $i=16$ & $i=4$ & & $i=16$ & $i=4$ \\
\hline $13-4$ & 0.0132 & 0.064 & 0.071 & & 0.660 & 0.695 \\
$14-3$ & 0.0118 & 0.063 & 0.061 & & 0.726 & 0.695 \\
$15-2$ & 0.0113 & 0.063 & 0.053 & & 0.771 & 0.695 \\
\hline \hline
\end{tabular}

and 15-2 the opposite is true: In both cases the best partition is found at the second level, $i=16$, and the best flow in terms of $\rho_{c}$ is found when giving information about the same level. In other words the two coincide. This means that if communities are organized in a hierarchical fashion, it is always best to give information at the level where the maximum modularity is found.

\section{CONCLUSIONS}

In this paper we have taken advantage of a model incorporating search and congestion simultaneously to investigate the impact that community structure has on information transport. We have shown that transport is compromised when community structure is introduced in the network since community structure implies the presence of bottlenecks. In fact, the better defined the communities are, the more affected packet transport becomes. We have also shown that transport can be dramatically improved by providing packets with information about the community structure. Finally we have shown that the largest improvements are found when the partition with the largest modularity is used to provide the information.

This suggests that it is possible to infer a priori what kind of information should be given to packets to optimize packet transport, just by identifying the community structure. By finding the communities at the level of highest modularity, and providing information at this level, packet transport appears to be optimal. The question remains: Is this always the case? It certainly seems possible to improve information transfer on an arbitrary network just by providing the search algorithm information about the community structure at the level of highest modularity. Since maximizing the modularity measure is $\mathrm{NP}^{1}$ hard [34], all community detection algorithms that depend on maximizing modularity are heuristic approximations and as such different identification algorithms find different partitions with varying values of optimal modularity for real networks. The results here suggest that giving information about the community structure as found by the most accurate algorithms would be best. But this remains to be shown in the case of real networks.

\footnotetext{
${ }^{1}$ In computational complexity theory, non-deterministic polynomial time (NP) is the set of decision problems solvable in polynomial time on a non-deterministic Turing machine. NP-hard problems are at least as hard as the most difficult problems in NP.
} 
[1] S. Boccaletti, V. Latora, Y. Moreno, V. Chavez, and D.-U. Hwang, Phys. Rep. 424, 175 (2006).

[2] J. Kleinberg, Nature (London) 406, 845 (2000).

[3] H. P. Thadakamalla, R. Albert, and S. R. T. Kumara, New J. Phys. 9, 190 (2007).

[4] L. A. Adamic, R. M. Lukose, A. R. Puniyani, and B. A. Huberman, Phys. Rev. E 64, 046135 (2001).

[5] V. Jacobson, Proceedings of SIGCOMM (ACM, Stanford, CA, 1988).

[6] T. Ohira and R. Sawatari, Phys. Rev. E 58, 193 (1998).

[7] A. Tretyakov, H. Takayasu, and M. Takayasu, Physica A 253, 315 (1998).

[8] A. Arenas, A. Diaz-Guilera, and R. Guimera, Phys. Rev. Lett. 86, 3196 (2001).

[9] R. Sole and S. Valverde, Physica A 289, 595 (2001).

[10] R. Guimerà, A. Arenas, A. Díaz-Guilera, and F. Giralt, Phys. Rev. E 66, 026704 (2002).

[11] B. Tadić, S. Thurner, and G. J. Rodgers, Phys. Rev. E 69, 036102 (2004).

[12] L. Zhao, Y.-C. Lai, K. Park, and N. Ye, Phys. Rev. E 71, 026125 (2005).

[13] M. Takayasu, H. Takayasu, and T. Sato, Physica A 233, 824 (1996).

[14] J. Duch and A. Arenas, Phys. Rev. Lett. 96, 218702 (2006).

[15] R. Guimerà, A. Arenas, and A. Díaz-Guilera, Physica A 299, 247 (2001).

[16] R. Guimerà, A. Díaz-Guilera, F. Vega-Redondo, A. Cabrales, and A. Arenas, Phys. Rev. Lett. 89, 248701 (2002).

[17] S. Sreenivasan, R. Cohen, E. Lopez, Z. Toroczkai, and H. E. Stanley, Phys. Rev. E 75, 036105 (2007).

[18] B. Tadić, G. J. Rogers, and S. Thurner, Int. J. Bifurcation Chaos (to be published).

[19] B. Danila, Y. Yu, J. A. Marsh, and K. E. Bassler, Phys. Rev. E
74, 046106 (2006).

[20] B. Danila, Y. Yu, S. Earl, J. A. Marsh, Z. Toroczkai, and K. E. Bassler, Phys. Rev. E 74, 046114 (2006).

[21] Z. Toroczkai and K. E. Bassler, Nature (London) 428, 716 (2004).

[22] P. Echenique, J. Gómez-Gardeñes, and Y. Moreno, Phys. Rev. E 70, 056105 (2004).

[23] M. Girvan and M. E. J. Newman, Proc. Natl. Acad. Sci. U.S.A. 99, 7821 (2002).

[24] J. Reichardt and S. Bornholdt, Phys. Rev. Lett. 93, 218701 (2004).

[25] R. Guimerà, S. Mossa, A. Turtschi, and L. A. N. Amaral, Proc. Natl. Acad. Sci. U.S.A. 102, 7794 (2005).

[26] M. E. J. Newman, Eur. Phys. J. B 38, 321 (2004).

[27] L. Danon, A. Dìaz-Guilera, J. Duch, and A. Arenas, J. Stat. Mech.: Theory Exp. 2005, P09008.

[28] A. Arenas, A. Diaz-Guilera, and C. J. Perez-Vicente, Phys. Rev. Lett. 96, 114102 (2006).

[29] A. Arenas, A. Diaz-Guilera, and C. J. Perez-Vicente, Physica D 224, 27 (2006).

[30] S. Fortunato and M. Barthélemy, Proc. Natl. Acad. Sci. U.S.A. 104, 36 (2007).

[31] M. Sales-Pardo, R. Guimera, A. Moreira, and L. Amaral, Proc. Natl. Acad. Sci. U.S.A. 104, 15224 (2007).

[32] R. Radner, Econometrica 61, 1109 (1993).

[33] M. E. J. Newman and M. Girvan, Phys. Rev. E 69, 026113 (2004).

[34] U. Brandes, D. Delling, M. Gaertler, R. Goerke, M. Hoefer, Z. Nikoloski, and D. Wagner, IEEE Trans. Knowl. Data Eng. 20(2), 172 (2008).

[35] The $Z_{\text {in }}=8$ corresponds to $Z_{\text {out }}=8$, at which point most community identification algorithms are able to detect the correct structure [27]. 